Chimia 46 (1992) 2I-25

(c) Schweiz. Chemiker-Verband; ISSN 0009-4293

\title{
Das Chemiestudium an der Ingenieurschule HTL Chur
}

\author{
Marcel Capaul'), Johann Forrer $\left.{ }^{2}\right)^{*}$, Heinrich Keller $^{3}$ ), Willi A. Ribi ${ }^{4}$ ), Klaus \\ Stalder ${ }^{5}$ ) und Bruno Wenk ${ }^{6}$ )
}

\section{Die Ingenieurschule HTL Chur}

Die Ingenieurschule HTL Chur wurde 1963 (damals als Abendtechnikum Chur) unter dem Patronat der Vereinigung Bündnerischer Wirtschaftsverbände gegründet. Man wollte die Abwanderung von Kadernachwuchs bremsen, die wirtschaftliche Entwicklung der peripheren Region fördern und tüchtigen Berufsleuten eine Weiterbildungsmöglichkeit öffnen. In Zusammenarbeit von privatwirtschaftlichen Unternehmungen, öffentlichen Verwaltungen, Gemeinden, Kanton und Bund hat sich eine Schulungsstätte entwickelt, die jährlich im Durchschnitt 50 HTL-Absolventen entlässt.

Die Ingenieurschule HTL Chur ist seit 1972 eine vom eidg. Volkswirtschaftsdepartement anerkannte Höhere Technische Lehranstalt im Sinne von Art. 59 des Bundesgesetzes über die Berufsbildung vom 19. April 1978. Die Schule wird vom Verein Ingenieurschule HTL Chur getragen; der Kanton Graubünden und die Stadt Chur sind im Vorstandsausschuss und im Schulrat vertreten.

\subsection{Studienangebot}

Die Ingenieurschule HTL Chur führt folgende Abteilungen:

a) Berufsbegleitende Schulen

- Architektur

- Bauingenieurwesen

\footnotetext{
*Korrespondenz: Dr. J. Forrer Ingenieurschule HTL Chur Ringstrasse 18 $\mathrm{CH}-7001$ Chur

') Dr. M. Capaul, Dozent für Technisch-Englisch

$\left.{ }^{2}\right)$ Dr. J. Forrer, Abteilungsleiter und Dozent für Organische Chemie

3) H. Keller, Dozent für Polymerchemie

$\left.{ }^{4}\right)$ Prof. Dr. W.A. Ribi, Direktor HTL Chur

5) K. Stalder, Dozent für Verfahrenstechnik

$\left.{ }^{6}\right)$ B. Wenk, Abteilungsleiter Telecom und Dozent für Informatik
}

- Chemie (einzige Ingenieurschule der Schweiz mit berufsbegleitendem Chemiestudium)

- Elektrotechnik (Energietechnik/Automation)

- Elektrotechnik (Nachrichtentechnik/ Informatik)

- Maschinenbau

b) Tagesschule

Erstmals in der Schweiz wird an unserer Schule ein Tagesstudium der Fachrichtung Telecom (Telekommunikation und Informatik) angeboten.

Die einzelnen Studienrichtungen werden durch Abteilungsleiter betreut. Über 100 nebenamtliche und 8 hauptamtliche Dozenten unterrichten die $c a$. 250 Studenten in max. 18 Klassen.

\subsection{Aufnahmebedingungen}

Seit Herbst 1990 führt die Ingenieurschule HTL Chur einen Vorbereitungskurs für sämtliche Studienrichtungen durch. Der einmal jährlich stattfindende Vorbereitungskurs umfasst 375 Lektionen, verteilt auf 25 Wochen zu je 15 Lektionen. Er ist berufsbegleitend. Es werden die Fächer Mathematik, Geometrie, Deutsch und Englisch erteilt. In jedem Fach sind 3-4 bewertbare Arbeiten für die Beurteilung des Absolventen notwendig. Wenn der Notendurchschnitt aller Fächer mind. die Note 4,5 erreicht, ist für den Teilnehmer ein prüfungsfreier Eintritt an unsere Schule möglich. Zukünftige Studenten, die den Vorkurs nicht absolvieren oder den Vorkurs nicht bestehen, haben die Möglichkeit, eine Aufnahmeprüfung zu absolvieren.

Prüfungsfrei zugelassen werden erfolgreiche Absolventen der BMS oder Mittelschüler mit abgeschlossener Matura und einer der Studienrichtung entsprechenden Berufspraxis.

\subsection{Studienablauf}

a) Berufsbegleitendes Studium

Das berufsbegleitende Studium dauert 9 Semester zu 20 Wochen mit maximum 22 Wochenlektionen. Der Student hat zudem einer Berufstätigkeit von mindestens 37 Stunden die Woche nachzugehen. Studienbeginn ist jeweils anfangs November und zwar im Zweijahresturnus. In den geraden Jahren beginnen Studien an den Abteilungen Elektrotechnik und Chemie; in den ungeraden Jahren die Studien für Architektur, Bauingenieurwesen und Maschinenbau.

\section{b) Tagesschule}

Das Tagesstudium dauert 6 Semester zu 20 Wochen mit max. 38 Lektionen pro Woche. Studienbeginn ist jeweils anfangs November im Jahresturnus.

\subsection{Schulgeld}

Das Semestergeld beträgt für alle Studienrichtungen zur Zeit Fr. 650.- exklusive Unterrichtsmaterialien.

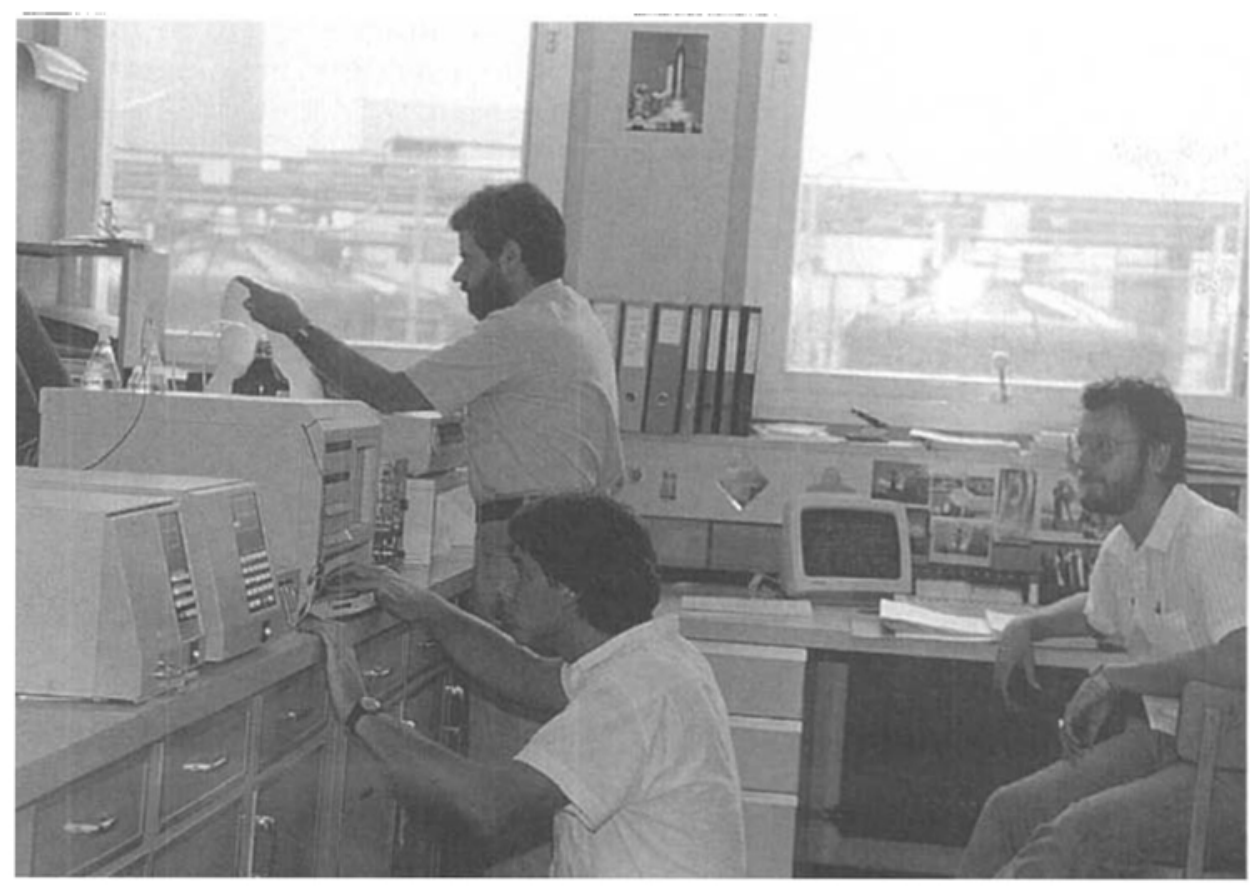




\subsection{Fachhörer}

Nach Möglichkeit werden zu einzelnen Vorlesungen Fachhörer, die sich über eine entsprechende Vorbildung ausweisen, zugelassen.

\subsection{Nachdiplomstudien/ Weiterbildungsmöglichkeiten}

Für die Weiterbildung der HTLAbsolventen bestehen an der Ingenieurschule HTL Chur folgende Möglichkeiten:

- diverse Nachdiplomstudien

- Fortbildungs- und Weiterbildungskurse

- höhere Fachkurse und Kaderseminarien

\section{Das Studium an der Abteilung Chemie}

Die Abteilung Chemie der Ingenieurschule HTL Chur ist etwas Besonderes: sie bietet als einzige in der Schweiz eine berufsbegleitende Ausbildung, welche in einem Lehrgang von 9 Semestern zur selbständigen Tätigkeit als Chemiker befähigt.

Die Ausbildung des HTL-Chemikers richtet sich grundsätzlich auf die praktischen, anwendenden Bereiche der Chemie. Diese Orientierung wird von unserer Schule in besonderem Masse verfolgt und realisiert durch folgende Grundsätze:

- Die Studierenden weisen bei Eintritt in die Schule eine mindestens 3jährige Praxis vor und müssen während des Studiums einer einschlägigen und dem Ausbildungsstand entsprechenden Berufstätigkeit nachgehen;

- Ein grosser Teil der Dozenten ist hauptberuflich in der Industrie tätig;

- Die Fachausbildung beinhaltet vorwiegend Aspekte und Fallbearbeitungen aus der Praxis.

Die berufsbegleitende Ausbildung sichert den Studenten einerseits die finanzielle Unabhängigkeit und bietet den Vorteil des direkten Kontaktes mit der Praxis fordert jedoch andererseits, dass sie den Hauptteil ihrer Freizeit der Ausbildung widmen. Während $41 / 2$ Jahren neben Berufsarbeit das anspruchsvolle Studium durchzustehen, verlangt vom Studierenden überdurchschnittliche Einsatzbereitschaft und Ausdauer gepaart mit einem entsprechenden Durchhaltewillen.

\subsection{Ausbildungsziel}

An unserer Abteilung für Chemie gilt der Grundsatz, dass keine Spezialisten ausgebildet werden. Wesentlich für die Vorbereitung des HTL-Chemikers auf seine Tätigkeiten im Berufsleben ist dabei die Vermittlung von soliden Grundlagen, welche es ihm gestatten rasch Spezialkenntnisse zu erwerben und sich in besondere Aufgabengebiete einzuarbeiten und zu vertiefen.

Die wichtigsten Zielsetzungen für die Ausbildung sind im Berufsbild des HTLChemikers enthalten, welches als Leitlinie an der Abteilung Chemie erarbeitet wurde.

\subsection{Berufsbild des HTL-Chemikers}

Die Ingenieurschule HTL Chur vermittelt ihren Chemie-Studenten die Grundlagen in allgemeinbildenden und mathematisch-naturwissenschaftlichen Fächern, die es ihnen ermöglichen, in verantwortlicher Stellung tätig zu sein. Gleichzeitig fördert sie persönliche Eigenschaften, die dazu nötig sind.

Im Vordergrund stehen Betriebsführung, Produkte- und Verfahrensentwicklung, Anlageplanung, Anwendungstechnik und Kontrollfunktionen im industriellen und öffentlichen Bereich.

Aufbauend auf praxisbezogene Grundlagen in Synthese und Analytik wird das Schwergewicht auf instrumentelle Methoden, Verfahrenstechnik und Polymertechnologie gelegt. Ausserdem wird die Bildung ökologischen Bewusstseins und die Schulung von Computeranwendungen in der Chemie gefördert.

\subsection{Die Ausbildung: Wege zum Studium, Lehrplan}

Voraussetzung für die Aufnahme als ordentlicher Student ist eine abgeschlossene Berufslehre als Chemielaborant und der erfolgreiche Besuch des von der Ingenieurschule Chur angebotenen Vorbereitungskurses, welcher für die Fächer Mathematik, Deutsch und Englisch das Eintrittsniveau für das HTL-Studium sichert. Für Mittelschüler mit oder ohne Maturität sowie für Absolventen einer Berufsmittelschule gelten Sonderregelungen.

Die 9semestrigen Chemie-Lehrgänge beginnen alle 2 Jahre. Während des 20 Wochen dauernden Semesters besuchen die Studenten neben der Berufsarbeit 22 Lektionen à 45 min pro Woche. Der theoretische Unterricht findet von Montag bis Freitag in den Abendstunden statt, während die Praktika tagsüber an Samstagen abgehalten werden. Während des Studiums stellt der Studierende den Stand des Könnens laufend unter Beweis: Semesternoten, Semesterarbeiten, Vordiplom-, Diplomprüfungen und Diplomarbeit sind Stationen, die den Fortschritt bis zum Abschluss bestätigen.

Die Chemie-Lehrgänge umfassen folgende Fachgruppen: allgemeinbildende Fächer, Chemie-Fächer, technische Fächer, Praktika.

Die Verteilung der einzelnen Fächer auf die Semester ist in der Tabelle wiedergegeben.

Die Gesamtzahl Lektionen ist mit 3860 tiefer als bei Tagestechnika mit ca. 4400 . Der Unterschied kommt durch eine geringere Anzahl von Praktikumslektionen zustande. Es wird hier bei den Mindestanforderungen des Eidg. Volkswirtschaftsdepartementes berücksichtigt und angerechnet, dass im berufsbegleitenden Studium der ständige Kontakt mit Chemiepraxis es sind z.Zt. 37 Wochenstunden Berufstätigkeit verlangt - gegeben ist.

\subsection{Unsere Absolventen}

Seit der Gründung der Ingenieurschule HTL Chur im Jahr 1963 wurden 12 Chemie-Kurse zu Ende geführt und 130 Studenten konnten bisher nach erfolgreich abgeschlossenem Studium das Diplom als HTL-Chemiker entgegennehmen. Es ist erfreulich berichten zu können, dass alle Absolventen in sehr verschiedenen Funktionen in der chemischen Industrie in Produktion, Entwicklung, Marketing, Umweltschutz, Sicherheit und auch im öffentlichen Dienst tätig sind und sich bewährt haben.

Welche Bedeutung für Graubünden der Möglichkeit des berufsbegleitenden Chemie-Studiums an der HTL Chur zukommt, wird durch die Statistik der Arbeitsorte unserer Absolventen belegt: $47 \%$ sind in GR, $13 \%$ in der Region SG/FL und $40 \%$ in der übrigen Schweiz in ihrem Beruf tätig.

\section{Gedanken zur Ausbildung und Erfahrungen im Unterricht}

\subsection{Mehr Fachwissen?}

Die Einsatzmöglichkeiten, die sich dem HTL-Chemiker bieten, sind sehr vielfailtig. Mit den Stichworten Produktion, Qualitätssicherung, Forschung und Entwicklung, Anwendungstechnik, Marketing, Verfahrensprojektierung, Umweltschutz/ Sicherheit und Informatik werden mögliche Einsatzbereiche mit verschiedenen Anforderungen genannt.

Der im Beruf stehende HTL-Chemiker wird sich jedoch rasch vertiefte fachliche Spezialkenntnisse aneignen müssen und wird feststellen, dass er während des Studiums in unterschiedlichem Ausmass für die Aufgaben des Berufs-Alltages vorbereitet wurde. Das ist sowohl für ihn als auch für die ausbildenden Lehrer an der Ingenieurschule HTL eine Herausforderung. Um den Bedürfnissen der Absolventen und der Industrie zu entsprechen, wurden Gespräche und Erhebungen in der Region GR, SG und FL durchgeführt.

Es hat sich ergeben, dass für die Beurteilung der Fähigkeiten nicht allein das Fachwissen entscheidend ist. Für den be- 
ruflichen Erfolg muss sich der Absolvent neben fachlichem Basiswissen und einer soliden Allgemeinbildung weitere Fähig-. keiten aneignen:

- Teamfähigkeit,

- Präsentationsfähigkeit,

- interdisziplinäre Arbeitsweise,

- Systematik und Intuition bei Problemlösungen.

Obwohl im berufsbegleitenden Studium diese Fähigkeiten im Chemiealltag ständig miterlebt werden, finden seit einigen Jahren an der Abteilung Chemie vermehrt Wege zu deren gezielter Aneignung Beachtung.

Der Unterricht und die Praktika werden nach Möglichkeit so gestaltet, dass mit Gruppenarbeiten, Teambildungen bei Semesterarbeiten, Vorträgen, Seminarübungen die zusätzlich zum Fachwissen notwendigen Fähigkeiten erkannt und geübt werden. Wir erwarten, dass die Absolventen mit diesen Erfahrungen aus dem Studium in ihrem Berufsleben den stets wachsenden Druck der Konfrontation mit neuen Aufgaben besser beherrschen werden.

\subsection{Technisches Englisch: ein wichtiges nicht zu unterschätzendes Unterrichtsfach}

Auf die Frage in obere Industrie-Etagen worauf bei der Ingenieur-Ausbildung besonders zu achten sei, hört man oft: sorgen sie dafür, dass ihre Absolventen Deutsch und Englisch beherrschen!

An einer berufsbegleitenden technischen Abendschule ist das kein leichtes Unterfangen. Geht es doch darum, an Stress und Zeitmangel, sowie an verständlicher Präferenz der technischen Belange nicht zu scheitern.

Den Weg zum Erfolg gibt es und dieser muss weg vom Vorurteil führen: 'Für allgemeinbildende Fächer keine Zeit!'.

Sprachbeherrschung bedeutet, sich als Ingenieur ausdrücken zu können, Gewandtheit in technischem Englisch vermittelt Selbstsicherheit, Brauchbarkeit in weltweitem Umfeld und sich verkaufen können sozusagen als 'homo technicus universalis'.

Der Schreibende macht als nebenberuflicher Dozent die Erfahrung stets von neuem, wie leicht es ist, die HTLStudenten vom unabdingbaren Stellenwert des Technisch-Englisch zu überzeugen. Es geht dann darum, die gewonnene innere Bereitschaft während der ganzen Unterrichtsdauer voll am Leben zu erhalten.

Dazu gilt es, von verschiedenen 'Rezepten' in optimaler Weise Gebrauch zu machen - angepasst an die Assimilationskraft der aktuellen Schülerschaft. Zu nennen sind:

Tabelle. Stundentafe

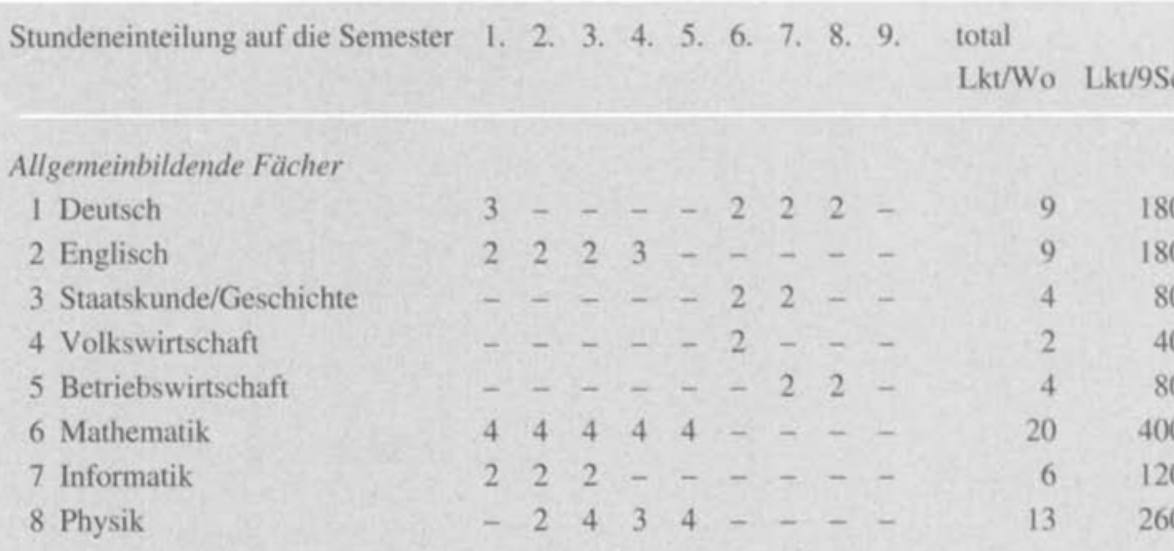

Chemie

9 Allgemeine Chemie

10 Anorganische Chemie

$42 \ldots \ldots+\ldots 6120$

11 Analytische Chemie

12 Instrumentelle Analytik

$-4-\ldots 480$

13 Physikalische Chemie

22

14 Organische Chemic

$-2-\ldots+240$

15 Ökologische Chemie

$\begin{array}{llll}2 & 2 & 2 & 3\end{array}$

$\begin{array}{llll}2 & 2 & 4\end{array}$

16 Technische Chemie

17 Farbstoffchemie

18 Polymerchemie

19 Polymertechnologie

Technische Fächer

20 Verfahrenstechnik

$-2,4$

2

260

21 Mess- und Regeltechnik

$-$

Praktika

22 Analytische Chemi

23 Physikalische Chemie

$66 \ldots \ldots+\ldots 200$

24 Instrumentelle Analytik

$-\begin{aligned} & 63-\ldots \\ & -33\end{aligned}$

25 Organische Chemie

$\begin{array}{lll}6 & 4 & 4\end{array}$

120

26 Farbstoffchemie

27 Polymertechnologie

28 Verfahrenstechnik

Lektionen pro Woche

$\begin{array}{llllllllll}21 & 22 & 22 & 22 & 21 & 22 & 22 & 21 & 20\end{array}$

193

Lektionen pro Semester

3860

- Aktuelles Vokabular mit entsprechenden Übungstexten:

Es ist bemerkenswert, wie wenig wirklich Berufsrelevantes, Modernes vorhanden ist. Die Sprachlehrer stammen selten aus dem Chemie- und IngenieurAlltag. Die Chemiker und Ingenieure sind nur ausnahmsweise Könner in spezialisiertem Sprachunterricht.

- Schaffen einer technischen Ambiance: Der Technisch-Englisch Student wird fiktiv z.B. als Montage-Ingenieur tausende von Kilometer weit weg vom Mutterhaus versetzt, da heisst es 'debrouillez-vous' mit den Einheimischen oder per Telefon oder Fax mit dem amerikanischen Lieferanten! Oder bei nächtlichem Störfall in einer Produktionsanlage unserer englischen Tochtergesellschaft; wie ist das Manual zu verstehen, wie kann das Belegschaftswissen mobilisiert werden?

- Einsatz von Sprachlabor: Besonders beim Basis-Englisch, später stärkeres Engagieren der Studenten in Kurzvorträgen, Diskussionen usw. mit der Möglichkeit von Profilierung oder Blamage!

- Unterricht unter Erwachsenen: Es gilt Gedächtnisstoff zu begrenzen und möglichst viel aus der Kombinati- 
on von sprachlichem und technischem Text-Zusammenhang herauszuholen. Kaum zu glauben, was hier Fremdwörter-Kenntnisse oder eine romanische Sprache für das technische Englisch helfen kann!

- Lernen für das Berufsleben:

Ein persönliches und unablässig mit Wichtigstem zu führendes 'pocket-size vocabulary' soll lebenslanges Vademecum werden und eine Verbindung vom Unterricht zur Berufskarriere sein.

- Sanfter Druck:

Ergibt sich z.B. durch Voraugenhalten des Schluss-Examens! Dabei wird eine Arbeit ohne Hilfsmittel im Unterricht Erarbeitetes beinhalten und eine andere mit erlaubten Hilfen den Umgang mit fremden Texten. So kommt sowohl der 'Fleissige' wie der 'Überlegene' zum Zuge.

Im 4semestrigen Englisch-Unterricht für Chemiker soll das Problem der ungleichen Sprachvorkenntnisse durch Vorkurse gemildert werden. Damit soll der Weg geebnet werden, schon vor dem EnglischAbschlussemester eigentliche technische Sprache vorzusehen. Diese umfasst Texte und Übungen zu Laborausrüstung und arbeit, Apparate und deren Gebrauch, Halbtechnik- und Grossanlage-Alltag, systemat. Texte zu organischer - und Polymer-Chemie, Patent-, Mathematik-, etwas technische Telefon- und Korrespondenz-Sprache.

\subsection{Informatik hält vermehrt Einzug}

\section{Motivation}

Ein Computer oder wenigstens ein Bildschirm und eine Tastatur, die mit einem zentralen Rechner verbunden sind, finden sich heute auch an den Arbeitsplätzen vieler Chemiker. Typische Anwendungen für den Rechnereinsatz sind z.B. das Verfassen von Berichten, die oft viele Grafiken enthalten, das Analysieren von Messwerten einer Versuchsreihe, die Simulation chemischer Reaktionen oder die Suche nach Fachliteratur in einer externen Datenbank.

Für den Chemiker ist der Computer vor allem ein Werkzeug, das ihm die rasche und exakte Ausführung gewisser Arbeiten erleichtert. Der Informatikunterricht an der Chemie-Abteilung einer Ingenieurschule HTL hat auf diese Gegebenheiten Rücksicht zu nehmen. Im Mittelpunkt des Informatik-Unterrichts steht deshalb die Vermittlung der Kenntnisse und das Üben der Fähigkeiten, die für einen effizienten Umgang mit dem Computer am Arbeitsplatz nötig sind. Selbstverständlich sind dabei die allgemeingültigen Grundlagen und Konzepte des Rechner- einsatzes zu behandeln und nicht herstellerspezifische Details.

Hin und wieder wird aber auch der Chemiker mit Informatikentwicklungsprojekten konfrontiert werden. Sei es, dass er als Mitglied einer Projektgruppe die Chemie-Fachkenntnisse, die zur Entwicklung der computergestützten Steuerung eines verfahrenstechnischen Prozesses nötig sind, einbringen muss, sei es, dass er sogar ein kurzes Spezialprogramm zur Untersuchung einer Messwertreihe nach einer neuen Analysemethode selbst entwickeln soll. In diesen Fällen muss der Chemiker die wesentlichen Grundlagen der professionellen Softwareentwicklung kennen, vor allem, um die im Projekt zu erwartenden Schwierigkeiten realistisch abschätzen und in der Planung berücksichtigen zu können. Diese Anforderungen bedingen, dass im Informatikunterricht an einer Chemie-Abteilung auch die Konzepte der strukturierten Programmierung diskutiert werden.

\section{Lehrinhalte}

Der Informatik-Unterricht an der Abteilung Chemie der Ingenieurschule HTL Chur beginnt bereits im 1. Semester. Er umfasst drei Semester zu je 2 Lektionen pro Woche, also insgesamt 120 Lektionen.

Im ersten Semester und in der ersten Hälfte des zweiten Semesters befassen sich die Studierenden mit dem Aufbau und der Funktionsweise von Arbeitsplatzrechnem und deren Betriebsystemen. Die grundlegenden Konzepte von Standardanwenderprogrammen(Textverarbeitung, Tabellenkalkulation, Präsentationsgrafik, Datenbanken) werden besprochen, und das Arbeiten mit derartigen Programmen anhand von Beispielen geübt. Im Sommersemester 1991 erfassten die Studierenden beispielsweise in Zweiergruppen über einen längeren Zeitraum die Zeiten, die sie für ihre berufliche Tätigkeit an Arbeitsplatz, für die Vorbereitung auf den Unterricht an der Ingenieurschule, für den Unterricht selbst, für die Bearbeitung von Hausaufgaben, für die Freizeit, usw. aufwendeten. Diese Daten wurden mit einem Tabellenkalkulationsprogramm ausgewertet und die Resultate mit einem Grafikprogramm anschaulich dargestellt. Ein Textverarbeitungsprogramm diente anschliessend dazu, einen Bericht über die durchgeführten Aufzeichnungen, die Auswertungen und die daraus gezogenen Schlüsse zu verfassen.

Am Ende des zweiten und im dritten Semester setzen sich die Studierenden mit der Programmierung eigener kleinerer Programme auseinander. Dazu wird die Programmiersprache Pascal verwendet, welche sich besonders gut zur anschauli- chen Darstellung der Konzepte der strukturierten Programmierung eignet. Sobald die Studierenden in der Lage sind, einfache Programme selbständig zu realisieren, wird ein gemeinsames Softwareentwicklungsprojekt in Angriff genommen. Damit lernen die Studierenden die besonderen Anforderungen an die exakte Formulierung eines Pflichtenheftes und der Schnittstellen zwischen den einzelnen Programmteilen kennen, wie sie bei der arbeitsteiligen Programmentwicklung im Team unabdingbar ist. Im Wintersemester 1991/92 hat dieses Projekt beispielsweise zum Ziel, mit einem 'personal computer' das Modell eines technischen Prozesses zu steuern, der die Entnahme von Paketen aus einem Warenlager, deren automatische Beschriftung mit Produkt- und Empfängerangaben und deren Weitergabe an die Spedition simuliert.

Am Ende des dritten Semesters findet der Informatik-Unterricht mit der schriftlichen Vordiplomprüfung seinen vorläufigen Abschluss. Die erarbeiteten Kenntnisse sind den Studierenden aber auch weiterhin für Arbeiten in anderen Fächern nützlich und werden später gegebenenfalls wieder zur Durchführung von Semester- und Diplomarbeiten benötigt.

\subsection{Ohne Verfahrenstechnik geht es nicht}

Unser Lernziel lautet in trockener Formulierung: 'Der Student kann physikalische Gesetze auf Einheitsoperationen übertragen, einfache verfahrenstechnische Vorgänge beurteilen und berechnen.'

Der Chemiker HTL gilt allgemein als der Betriebsleiter in der chemischen Industrie. Das Berufsbild unserer Schule ist etwas differenzierter: es stellt neben Betriebsführung und Anwendungstechnik ausdrücklich die Verfahrenstechnik und die Anlagenplanung mit in den Vordergrund. Tatsächlich ist eine ganze Reihe von Absolventen unserer Abteilung erfolgreich in diesen Berufszweigen tätig.

Dies bleibt nicht ohne Konsequenzen für das Studienfach Verfahrenstechnik. Wir arbeiten ausgesprochen praxisbezogen und bleiben immer im Gesamtrahmen der Planung verfahrenstechnischer Grossanlagen. Unsere Berechnungs- und Praktikumsbeispiele sind, wie auch die Diplomarbeiten, grossenteils der aktuellen Berufstätigkeit der Dozenten entnommen. Natürlich sind dabei - mit Rücksicht auf didaktische Bedürfnisse - Kompromisse einzugehen.

Die Verfahrenstechnik an der ChemieAbteilung ist mit insgesamt 260 Unterrichts- und 160 Praktikumslektionen, verteilt auf vier Semester, recht grosszügig dotiert. Es muss aber berücksichtigt werden, dass der Chemie-Student an unserer 
Schule weder Strömungslehre noch technische Thermodynamik als separates Grundlagenfach belegt. Die notwendigen Grundkenntnisse in diesen Disziplinen sind daher in den Lernstoff der Verfahrenstechnik integriert. Dementsprechend werden mechanische Einheitsoperationen eingehend behandelt. Dies hat sich im Laufe der Zeit als sehr zweckmässig erwiesen, stehen doch dem Chemie-Studenten auf Grund seines Ausbildungsganges die thermischen Trennoperationen um einiges näher. Eindampfen, Kristallisieren, Destillieren und Rektifizieren werden daher im theoretischen Unterricht recht knapp behandelt, eine gewisse Kompensation erfolgt im Praktikum.

Besonderes Gewicht legen wir auf numerisches Rechnen. Die 'Rechnergläubigkeit' und -abhängigkeit der jüngeren Generation hat bewirkt, dass das Gefühl für die Grössenordnung von physikalischen Werten abhanden gekommen ist. Schätzen und Kopfrechnen sind ausgesprochene Schwachpunkte unserer Studenten; sie gehen einher mit dem Problem des Chemikers im Umgang mit Masseinheiten.

Wir erarbeiten daher den Lernstoff zu einem guten Teil anhand von Rechenbeispielen sowie von Fragen der Studenten. Die physikalischen Grundlagen werden im Skriptum dargestellt und im Unterricht in knapper Form erläutert. Unsere Studenten lösen in 4 Semestern mindestens 200 Übungs- und Prüfungsaufgaben. Weil die Lücke zwischen Taschenrechnern, Tabellenkalkulationsprogrammen und den marktüblichen leistungsfähigen, aber schwer zu beherrschenden Modellierprogrammen relativ breit ist, leiten wir die Studenten auch dazu an, gängige Berechnungsformeln und -methoden in lauffähige PC-Programme umzusetzen. Dabei entstehen oft recht originelle, aber dennoch durchaus zweckmässige Hilfsmittel.

Die Statistik zeigt, dass 20-25\% der Studenten ihre Diplomarbeit in Verfahrenstechnik ablegen. Die Risiken, die sich beim Arbeiten an unbekannten Pilotanlagen ergeben, nehmen wir bewusst in Kauf. In den über 25 Jahren des Bestehens unserer Schule ist in diesem Fach noch kein einziger Diplomand aus versuchstechnischen Gründen 'abgestürzt'.

\subsection{Anspruchsvolle Zielsetzung in Poly- merchemie und Polymertechnologie}

Dem Leitbild der HTL entsprechend steht nicht die Ausbildung zum Spezialisten im makromolekularen Bereich im Vordergrund; vielmehr sollen erweiterte Kenntnisse in polymeren Belangen den HTL-Absolventen zur raschen Einarbeitung in Entwicklung, Produktion, Anwendungstechnik oder Verarbeitung von Po-

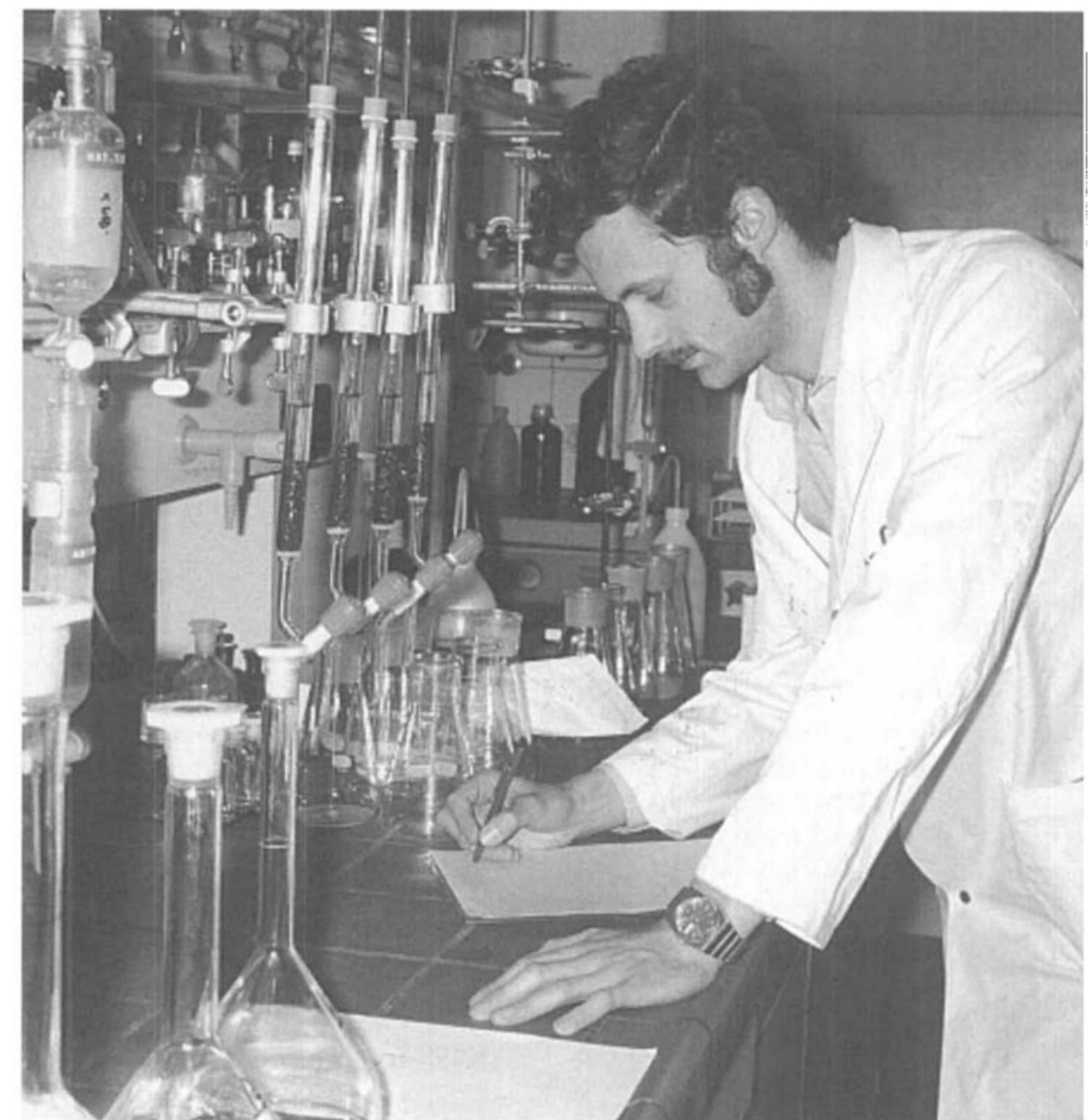

lymeren als Kunst- und Faserstoffe befähigen.

Die praxisorientierte Ausbildung, die nach neu konzipiertem Lehrplan ausgerichtet ist, bringt nach der vorliegenden Erfahrung die Voraussetzungen zur Erfüllung der anspruchsvollen Zielsetzung in den Fächern:

\section{Polymer-Chemie}

(1 1/2 Semester mit total 60 Lektionen) Grundlagen der Synthese und Reaktionen polymerer Substanzen mit Modifikationsmöglichkeiten zur Eigenschaftsbeeinflussung

\section{Polymer-Technologie}

( $11 / 2$ Semester mit total 60 Lektionen)

Technik in Produktion und Anwendung polymerer Rohstoffe unter besonderer Berücksichtigung der chemischen und physikalischen Charakteristik

Besondere Bedeutung kommt dem polymertechnologischen Praktikum (total 160 Lektionen) zu, wobei neben schulungsmässigen Laborübungen in Arbeitsgruppen spezielle Themata in Analytik, Nachbehandlung und Veredlung mit praxisbewährten Rezepten oder ausgewählten Problemstellungen bearbeitet werden. Es stehen dabei Geräte und Einrichtungen von der Lehranstalt nahestehender Firmen mit zur Verfügung. In besonderen Fällen erfolgt die Betreuung zusätzlich durch Fachpersonal.

Durch Exkursionen in Polymere herstellende oder verarbeitende Betriebe werden die im Unterricht vermittelten theoretischen Kenntnisse massgeblich vertieft und in Seminarien die Formulierung und Präsentation von Sachverhalten und $\mathrm{Be}$ urteilungen z.T. komplex gelagerter $\mathrm{Zu}$ sammenhänge geschult.

Etwa 10-15\% der Absolventen diplomieren mit einer Arbeit auf polymerem Gebiet und mit Anerkennung durch neutrale Experten aus anderen Lehranstalten oder der Industrie. Eine beträchtliche Anzahl unserer Absolventen sind in Entwicklung, Produktion, Anwendungsund Verarbeitungstechnik als qualifizierte Mitarbeiter und z.T. in Kaderstellung tätig. 